\title{
Mobile Application Development with Android
}

\author{
Rajesh Keshavrao Deshmukh, Shilpa Markandey, Pooja Sahu
}

Department of CSE, SSIPMT, Old Dhamtari Road, Sejabahar P.O., Mujgahan, Raipur, Chhattisgarh 492015, India

\begin{tabular}{l}
\hline Article Info \\
\hline Article history: \\
Received Feb 9, 2018 \\
Revised Jul 9, 2018 \\
Accepted Aug 11, 2018 \\
\hline
\end{tabular}

Keyword:

Android Architecture

Security

Version

\begin{abstract}
The Android is mobile platform. It is an open source and free operating system application, by Google it is developed and maintained. It was designed essentially for touch screen mobile devices, such as and tablet, computers, smart phones, watch television, cars etc. Android is one of the most widely used mobile OS. Android is a not only operating system but also key applications and middleware. Android is an open source operating system. It is developed by the open handset Alliance, led by Google, and other companies. Those are used to android studio 2.2.3 version and development the mobile application.
\end{abstract}

\section{Corresponding Author:}

Rajesh Keshavrao Deshmukh, Department of CSE,

SSIPMT,

Old Dhamtari Road, Sejabahar P.O., Mujgahan, Raipur, Chhattisgarh 492015, India.

Email: r.deshmukh@ssipmt.com

\section{INTRODUCTION}

The Android is technology. It is open source operating system, which means that anyone who wants to use Android can do so by downloading the full Android source code. That was used for development mobile based application and desktop based application. This is complete set of software for mobile device such us tablet computer ,smart phone, notebooks, watch and book readers, set-top boxes etc. it content three things Operating system, middleware, key apps. The android is Linux based operating system. More than 30 companies working to open up the mobile handset environment. Mobile application development is very good demand in market. The application are usually development the java language using the android development tools .the development tools are available in market such as native development kit for application or extension in $\mathrm{C}$ or $\mathrm{C}++$, Google app inventor etc. It can run on many different devices from many different manufacturers. The android is powerful and very popular operating system it can supports large number of applications in touch screen Phone. The hardware supports android software which is based on ARM architecture platform. These applications are more comfort Zone and advance Level for the users. The android is an open source operating system that means it's free and users can easily access and use it the android application. The android has got millions of apps available. The android help us to manage our life one or other way and the application are available in market low cost at that reasons android is very popular in world.

\section{HISTORY}

The world most popular mobile operating system launch in 2003 Initially, android incorporation founded by Andy Rubin in Palo Alto, California, United States in October, 2003. In 17th August 2005, Google purchase android Incorporation. Since then, android become subsidiary of Google Incorporation. Rich Miner, Andy Rubin, Nick Sears and Chris White is the key employees of Android Incorporation. At that time camera 
is more expensive to buy so that android shifted camera into smart phones. Andy Rubin love Robots so that he given the nick name of Android is coworkers. In 2007, Google announces the development of android OS. In 2008, HTC launched the first android mobile.

\subsection{Android versions:}

There are Many Versions of android but Versions 1.0 and 1.1 were not released under specific codename

Table 1. Versions of Android

\begin{tabular}{cccc}
\hline Version & Code name & API level & Release date \\
\hline 1.0 & No codename & 1 & September 23, \\
1.1 & No codename & 2 & February 9, 2009 \\
1.5 & Cupcake & 3 & April 27, 2009 \\
1.6 & Donut & 4 & September 15, \\
2.1 & Éclair & $5-7$ & October 26, 2009 \\
2.2 & Froyo & 8 & May 20,2010 \\
2.3 & Gingerbread & $9-10$ & December 6,2010 \\
$3.1-3.3$ & Honeycomb & $11-13$ & February 22,2011 \\
4.0 & Ice Cream Sandwich & $14-15$ & October 18,2011 \\
$4.1-4.2-4.3$ & Jelly Bean & $16-18$ & July 9, \\
4.4 & KitKat & $19-20$ & October 312013 \\
5.0 & Lollipop & $21-22$ & November 12,2014 \\
6.0 & Marshmallow & 23 & October 5,2015 \\
7.0 & Nougat & $24-24$ & August 22,2016 \\
$8.0-8.1$ & Oreo & $26-27$ & August 21,2017 \\
\hline & & &
\end{tabular}

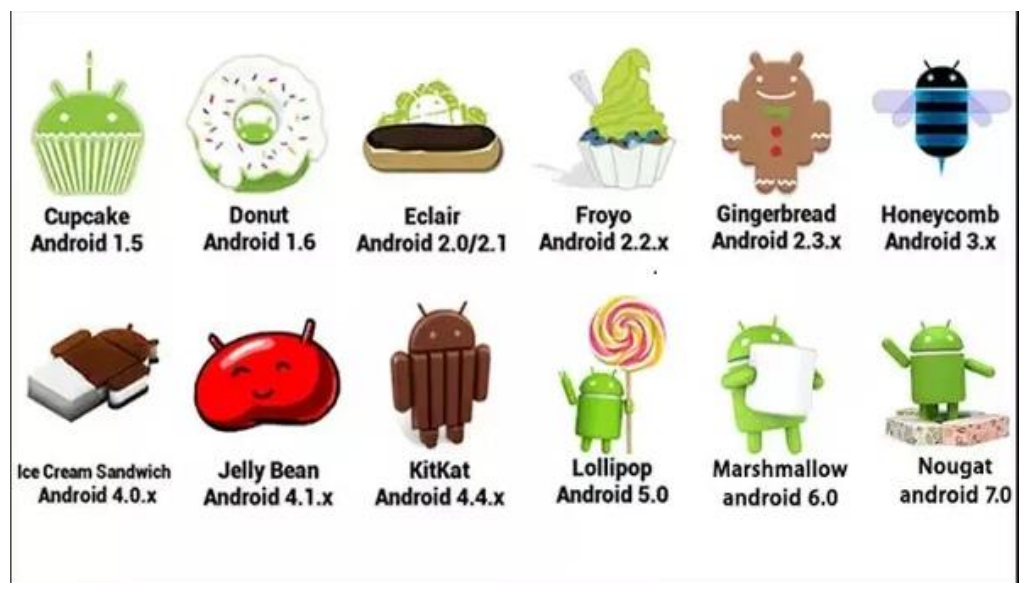

Figure 1. Of Android Versions

\section{FEATURE OF ANDROID}

- Storage: A lightweight relational database SQLite, for purposes of data storage it is used.

- Connectivity: It support many connectivity technology like WIFI, BLUETOOTH, UMTS, WIXMAX, CDMA, and GSM/EDGH.

- $\quad$ Messaging: The android is support feature of the both MMS and SMS.

- Web browser: Coupled with Chrome's V8 JavaScript engine supporting CSS3MMS and HTML5, based on the open-source Web Kit layout engine.

- $\quad$ Multi-touch: Android have a feature of Multitouch which is first made by handset HTC Hero. 
- $\quad$ Multi-tasking: Various application can run simultaneously. The user can jump from one task to another task at the same time.

- $\quad$ Tethering: The android is Supports sharing of Internet connections as a wired/wireless hotspot.

\section{ANDROID ARCHITECTURE}

The software stack is spilt into five layer:

- The application layer

- The application frame works

- The libraries

- The runtime

- $\quad$ The kernel

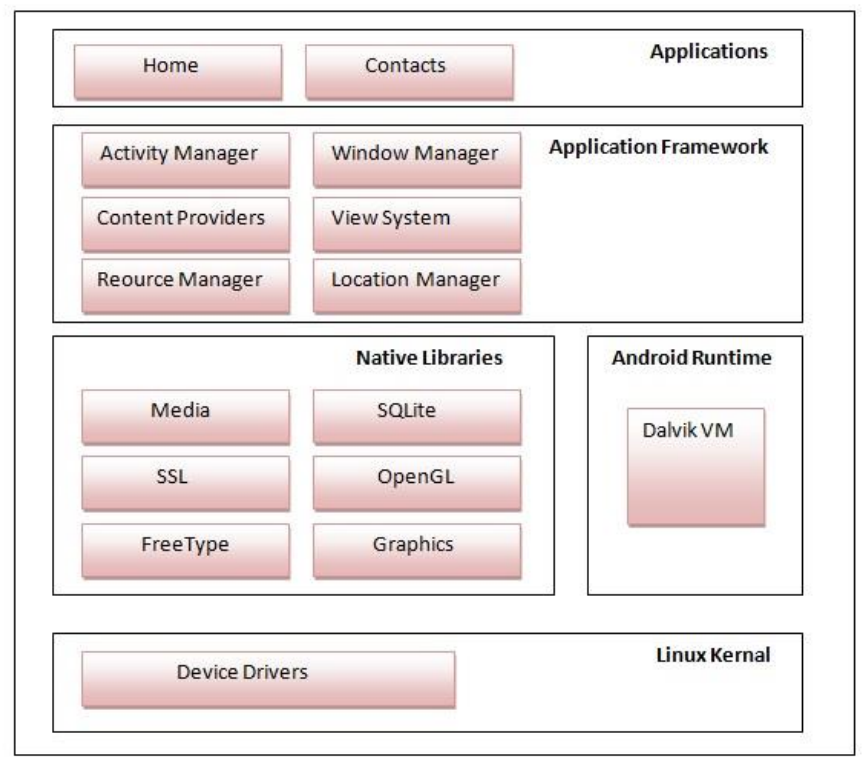

Figure 2. Android Architecture

\subsection{The Kernel}

The android is based on Linux 2.6 kernel architecture. This layer is core of android architecture. It provides service like processor and power management, security, memory management etc.

\subsection{The Native Libraries}

There Android has its own libraries, which is written in $\mathrm{C} / \mathrm{C}++$. These libraries cannot be accessed directly. Using application framework we can access these libraries. There are libraries which help to access many type of web browsers for this there is a web library and libraries for video and android formats etc.

\subsection{The Android Run Time}

The Android Runtime is an application. Which is run time environment .It is used by operating of android. Where you have limited battery, limited CPU, limited memory. Android have its own virtual machine is called Dalvik. It is run on android application. Dalvik is used in mobile devices such as tablets, phone etc. Program are commonly written in java and compile to byte code.

\subsubsection{Android Run Time}

This is in blue meaning that it's written in the java programming language. The internal library contains all of the collection utilities, class, IO and all the utilities.

\subsection{Appication Framework}

The application framework is the toolkit that all application use and this is all written in java programming language These applications include the ones that come with a phone like the phone application, 
or home applications, .It includes application written by Google, and includes apps that will be written by you. Every application use same APIs and framework.

\section{These are as follows:}

- Activity manger: the manages the lifecycle of application. It enables proper management of all activities. All the activities are controlled by manager.

- $\quad$ Resource manager: it provide access to non-code resources such as graphic etc.

- Location manager: if fires alerts when user enter or leaves a specified geographical location

- Package manager: it is use to retrieve the data about installed packages on device.

- Window manager: it is use to create view and layouts.

- Telephony manager: it is use to handle setting of network connection and all information about services on device.

\subsection{Application Layer}

Application layer is in the top layer. In this layer sharing of data and transfer of files. It involves the contacts application, the home application, apps and the browser. It is the top layer in android architecture. All the native application are like Google maps, camera, SMS, browser, calendars, and contacts are. With the help of application of framework to operate this application work with end user

\section{SECURITY}

All Android is design more technical security features and simply presented to user that means they can be easily controlled through the interface. To improving your Android device's security this method is straightforward methods can include: using a pin or fingerprint, password, setting your phone to lock after a period of inactivity, only enabling wireless connections that you use, and only installing Android apps you believe and have personally cheked.

Google allows Android applications into its marketplace and this application are tested and proven, secure by Google, it means that the user will be protected and less of a chance of installing virus and malicious app. For installation of an application, Android security system prompts the user to allow this installation. Meaning that it is impossible to remotely install and run an application. By regularly installing system updates users can secure their android devices from attackers.

Android is a multi-process system in which each application (and part of the system) runs in its own process. The security between the system and application is getting through standard Linux facilities, such as group IDs and user that are assigned to the applications. When attackers attack on device, android platform help to protect the smart from attackers.

There are key components of android security which are described as follows:

- Design review: by the developers design of security model is reviewed. So that while using this model the risk level will be very less.

- Code review and penetrating testing: the main aim of code review is that it will be test that how the system will become powerful?

- $\quad$ Open source and community review: android have open source and community review such as Linux kernel.

\section{ADVANTAGE AND DISADVANTAGE OF ANDROIDS}

Many advantage or disadvantage of android application.

\subsection{Advantages of Android}

- Android phone is easy to access.

- It is the easy to carry.

- It is more secure.

- With the support of many application, the user can change the screen display.

- It is platform independent.

- The major advantage is android is multitasking.

- It is support the Google service.

- It given you better notification.

- A more mature platform. 


\subsection{Disadvantage of Android}

- Some time attackers can attack android.

- Android operating system having lot of process because of this android OS hang.

- If memory is full then android phone getting slow.

- Extremely inconsistence in design among apps.

- It is unstable and some time crash.

\section{CONCLUSION AND FUTURE SCOPE}

Android is open source platform it can easily development the mobile based application All the APIs are available in order to develop these applications. It is very secure and its help to protect from attackers. Many version of android s available and latest version is Oreo 8.1. In the field of artificial intelligent many type of beneficial robot can be made which is require in future. Which is in progress to use in cars, watches and also Android TV.

\section{REFERENCES}

[1] Vikas Agrawal, Shruti Agrawal, Rajesh Deshmukh "Analysis and Review of Encryption and Decryption for Secure Communication" International Journal of Scientific Engineering and Research (IJSER) ISSN (Online): 2347-3878, Volume 2 Issue 2, February 2014.

[2] Li Ma, Lei Gu and Jin Wang "Research and Development of Mobile Application for Android Platform" International journal of Multimedia and Ubiquitous Engineering.

[3] https://www.javapoint.com/androoid-history-and-versions

[4] https://www.digitaltrends.com/mobile/android-version-history

[5] https://www.scribd.com/document/79741205/Paper-Presentation-on-Andriod

[6] http://www.android.com 